\title{
Monolayers and 3D films of cholesteryl derivatives at the air-water interface
}

\author{
P. Viswanath and K. A. Suresh* \\ Raman Research Institute, Sadashivanagar, Bangalore, 560 080, India.
}

\section{Supporting Information}

*Corresponding author. E-mail : suresh@rri.res.in. 


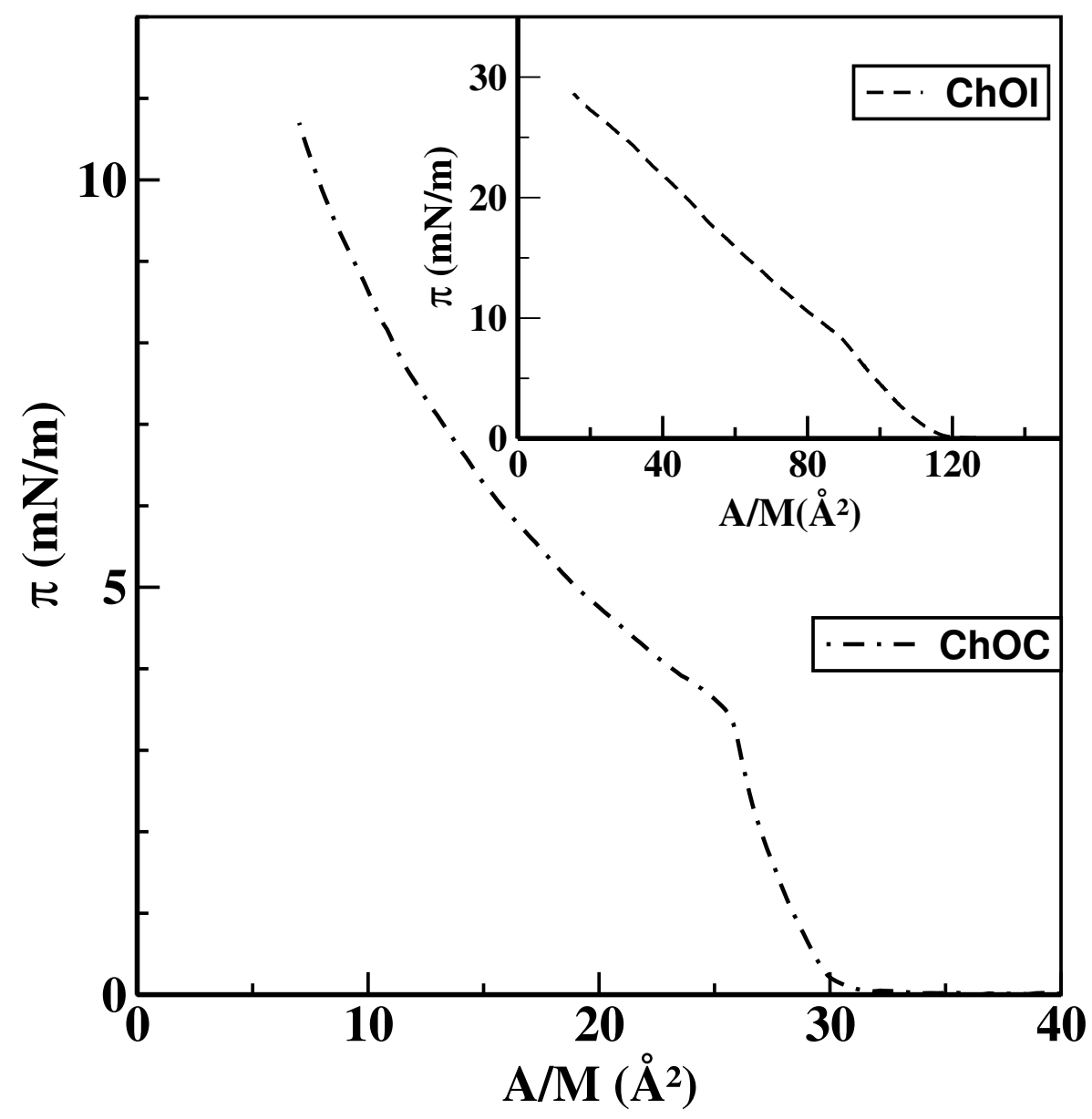

Figure S 1: Surface pressure $(\pi)$ - area per molecule $(A / M)$ of cholesteryl oleyl carbonate (ChOC) at $t=25{ }^{\circ} \mathrm{C}$ showing a collapse pressure of $3.4 \mathrm{mN} / \mathrm{m}$ with a limiting area per molecule of $28.7 \AA^{2}$. The inset shows the $\pi-A / M$ of cholesteryl oleate (ChOl) with a collapse pressure of $8.2 \mathrm{mN} / \mathrm{m}$ with limiting area per molecule of $114.3 \AA^{2}$.

Cholesteryl hydro cinnamate did not form a stable monolayer and gave rise to irreproducible isotherms. 


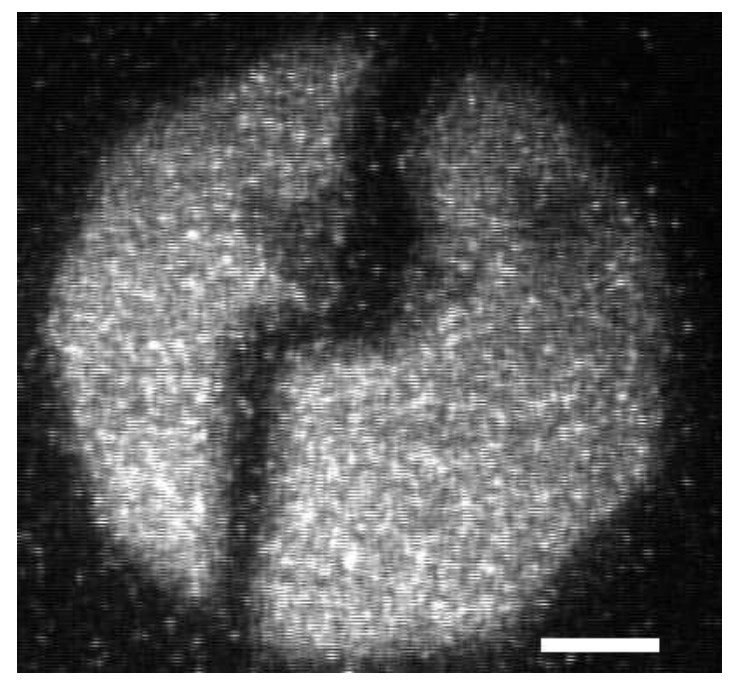

Figure S 2: Cholesteryl myristate image under epifluorescence microscope at an $A / M$ of $99.4 \AA^{2}$. Figure shows the presence of 3D structures with voids (gas phase). Scale bar represents $50 \mu \mathrm{m}$.

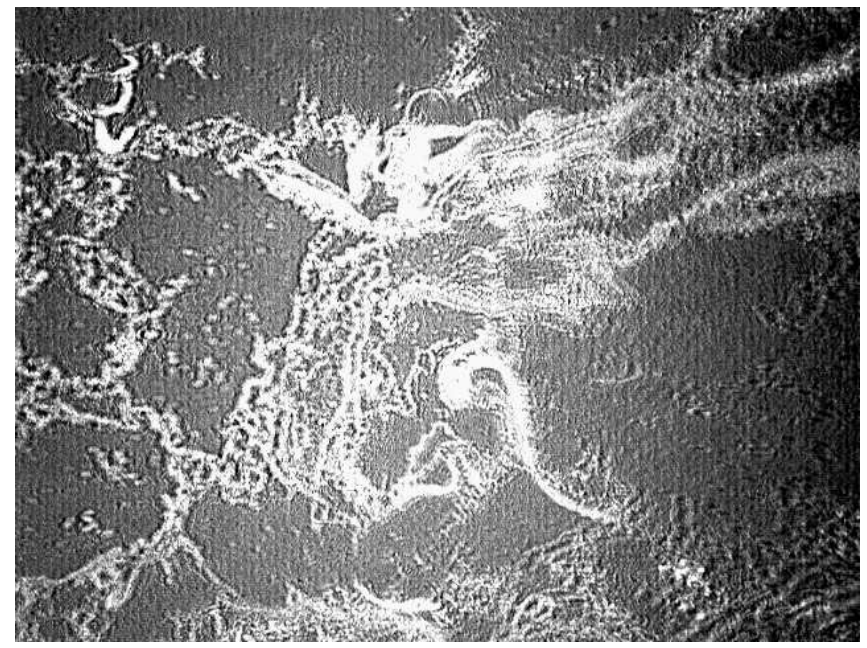

(a) $A / M=557.9 \AA^{2}$

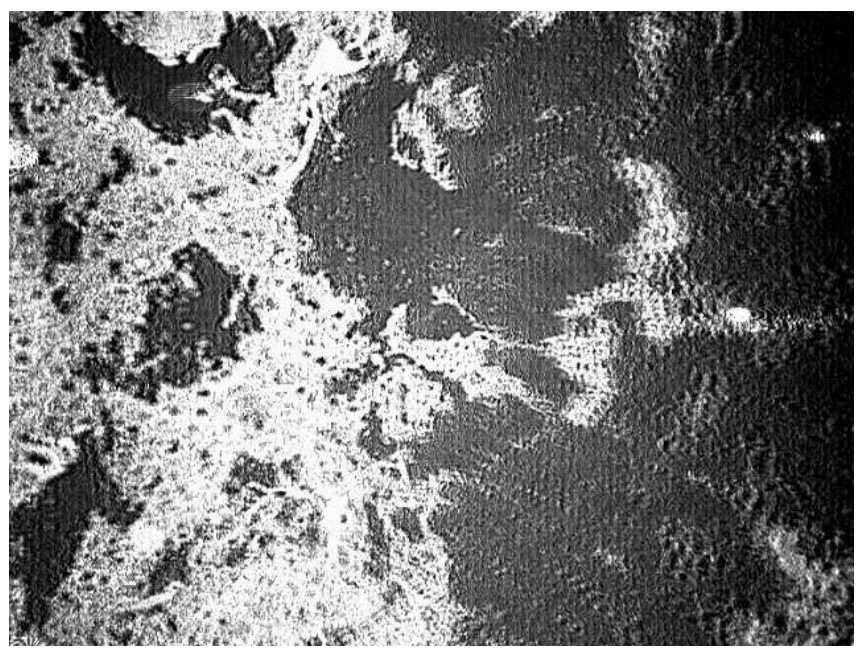

(b) $A / M=165.8 \AA^{2}$

Figure S 3: Brewster angle microscope images for cholesteryl palmitate seen at very low surface pressures $(\approx 0.1 \mathrm{mN} / \mathrm{m})$. Figure(a) shows the presence of highly irregular crystalline structure with voids (gas). Figure(b) shows the densely packed crystalline structure with less voids. Scale of each image is $6.4 \times 4.8 \mathrm{~mm}^{2}$. 


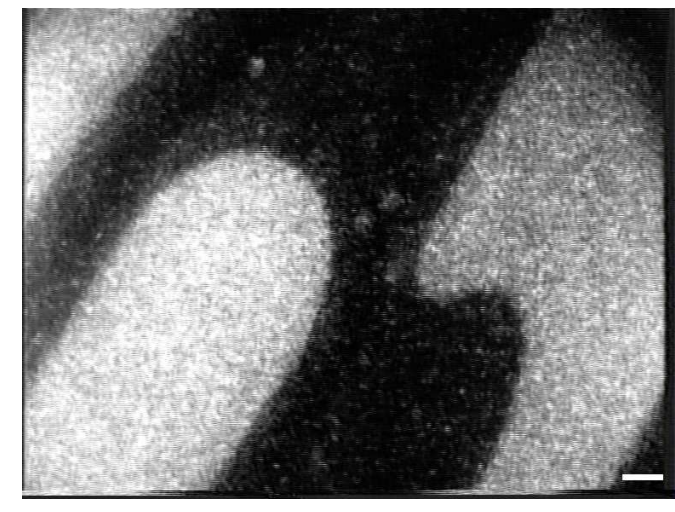

(a) $A / M=38 \AA^{2}$

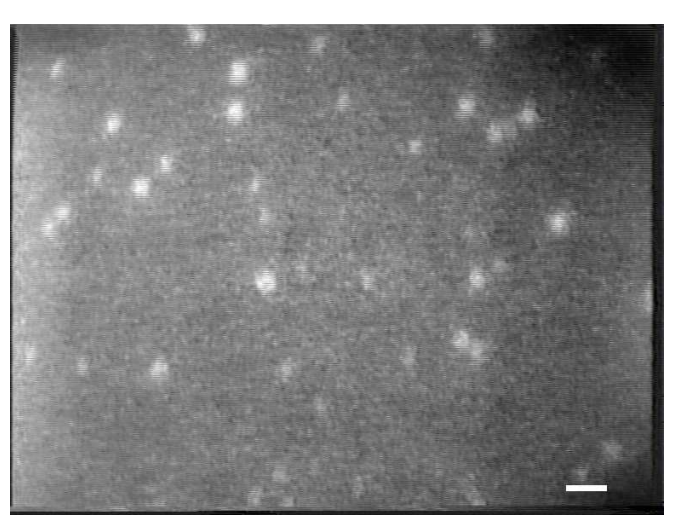

(c) $A / M=20.1 \AA^{2}$

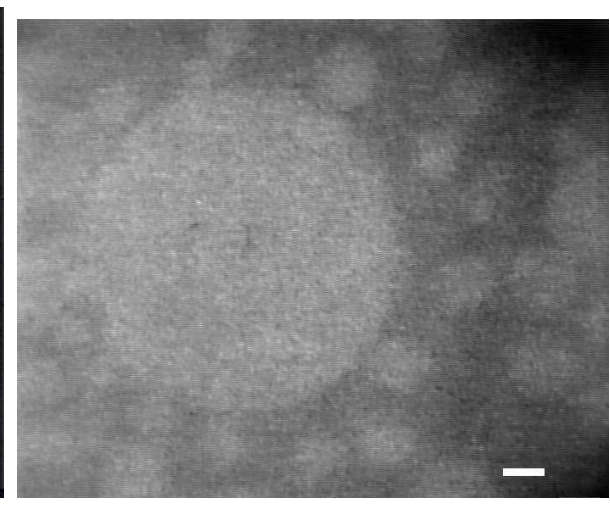

(b) $A / M=36 \AA^{2}$

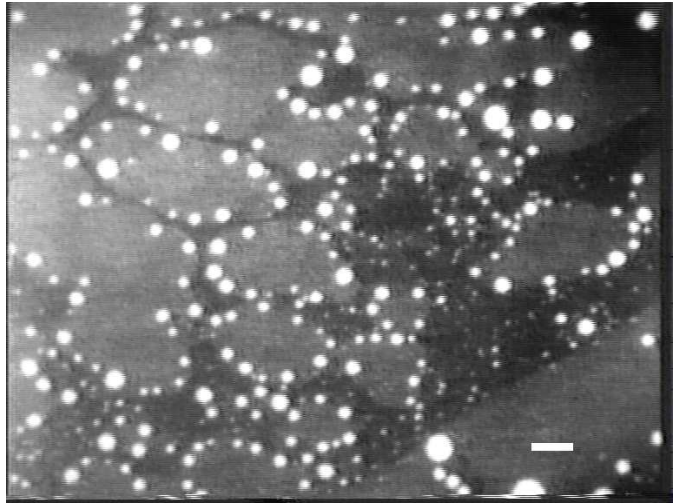

(d) $A / M=15.2 \AA^{2}$

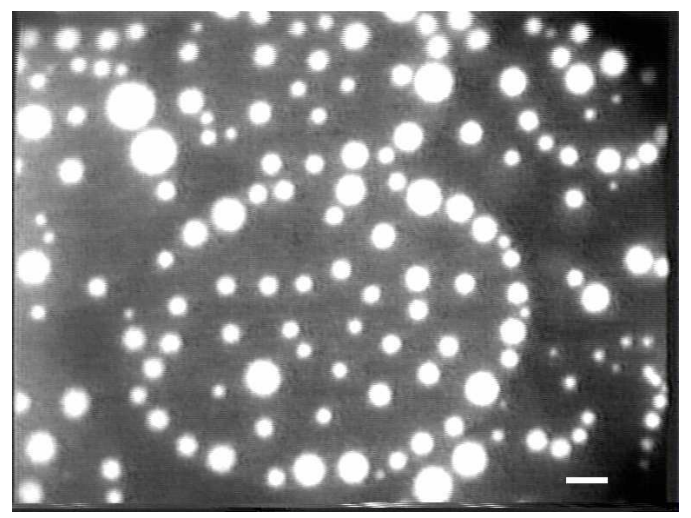

(e) $A / M=11.3 \AA^{2}$

Figure S 4: Cholesteryl oleyl carbonate images under epifluorescence microscope. In Figure(a), the coexisting $G$ (dark), $L_{1}^{\prime}$ (grey) and traces of $L_{1}$ (bright) phases are seen. Figure(b) shows the growth of circular $L_{1}$ domains which coexist with $L_{1}^{\prime}$ phase in the background. Figure(c) shows the collapsed state where brighter $3 \mathrm{D}$ dots coexist with $L_{1}$ phase (background). Figure(d) shows the domains of varying intensity coexisting with brighter dots. These brighter dots transform to circles. Figure(e) shows the domains of slightly different intensities coexisting with brighter 3D circular domains. These 3D circular domains arrange themselves near the periphery of the domains of varying intensity. Scale bar represents $50 \mu \mathrm{m}$. 